\title{
Significance of aldehyde dehydrogenase 1 expression in stromal cells of diffuse large B-cell lymphoma
}

\author{
SHIGEKI FUJITA, EIICHI MORII, NUR RAHADIANI, NAOKI WADA, \\ YUMIKO HORI, JUN-ICHIRO IKEDA and KATSUYUKI AOZASA
}

Department of Pathology, Osaka University Graduate School of Medicine, Suita 565-0871, Japan

Received March 13,2011; Accepted April 20, 2011

DOI: $10.3892 /$ etm.2011.269

\begin{abstract}
Aldehyde dehydrogenase (ALDH) 1 is expressed in various stem/progenitor cells, including cancer-initiating cells (CICs). In the present study, ALDH1 expression was immunohistochemically examined in diffuse large B-cell lymphoma (DLBCL). Unexpectedly, ALDH1 expression was occasionally detected in stromal cells, but not in lymphoma cells. Positively stained cells were $\mathrm{CD} 8^{+}$cells with voluminous cytoplasm and fascin ${ }^{+}$cells with reticular morphology, designated as macrophages and dendritic cells (DCs), respectively. The presence of $\mathrm{ALDH}^{+}$macrophages was not correlated with stage, response to therapies and prognosis. However, cases with $\mathrm{ALDH}^{+}$DCs were mostly nodal, which showed a poorer response to therapies and a worse overall survival rate than cases without $\mathrm{ALDH}^{+} \mathrm{DCs}$. The presence of $\mathrm{ALDH} 1^{+}$ DCs appears to be involved in the malignant potential of DLBCL in lymph node.
\end{abstract}

\section{Introduction}

Aldehyde dehydrogenase (ALDH) 1 is a predominant isoform of the ALDH family in mammals, which oxidizes retinol to retinoic acid at the early stage of stem cell differentiation (1). ALDH1 is expressed in various stem/progenitor cells, such as hematopoietic and neural stem cells (1-3). In addition to normal tissues, a small population of tumor cells expresses ALDH1 at a high level. The ALDH1-high population of tumor cells is resistant to antitumor agents and exhibits a tumorigenic potential, suggesting that ALDH1 is one of the markers for cancer-initiating cells (CICs) (1). The presence of a large number of ALDH1-high cells is indicative of poor prognosis in cancers of the breast $(1,4)$, lung $(5)$, pancreas $(6)$, bladder $(7)$ and prostate (8).

Correspondence to: Dr Eiichi Morii, Department of Pathology, Graduate School of Medicine, Osaka University, Yamada-oka 2-2, Suita 565-0871, Japan

E-mail: morii@patho.med.osaka-u.ac.jp

Key words: aldehyde dehydrogenase 1, diffuse large B cell lymphoma, stroma, dendritic cells, macrophages, prognosis
CICs were first identified in leukemia (9). Although lymphoma is the most common disease among hematologic malignancies, CICs have not yet been determined in lymphoma. In the present study, ALDH1 expression was examined in tissues involved by diffuse large B-cell lymphoma (DLBCL) to investigate the presence of CICs. Unexpectedly, no lymphoma cells expressed ALDH1. Instead, ALDH1 expression was detected in surrounding non-tumorigenic cells. Stromal cells consist of vasculature, fibroblasts and inflammatory cells, such as lymphocytes, macrophages and dendritic cells (DCs). It is well known that stromal cells affect the character of tumors (10). For example, the macrophage subpopulation named M1 produces interleukin (IL)-12 which promotes tumoricidal responses, whereas another subpopulation, named M2, produces IL-10 which aids in tumor progression (11). In the present study, ALDH1 expression in non-tumorigenic stromal cells of tissues involved by DLBCL was evaluated, and its clinical implications were examined.

\section{Materials and methods}

Clinical samples. Forty-six patients, who were diagnosed with DLBCL at Osaka University Hospital during the period from April 2006 to December 2009 and whose follow-up data could be accessed, were examined. Clinicopathological findings in these 46 patients are summarized in Table I. There were 19 men and 27 women with ages ranging from 29 to 78 years (median age, 63). Location of DLBCL was nodal in 25 cases and extranodal in 21 cases. Immunohistochemically, the large lymphoid cells were $\mathrm{CD} 20^{+}$and $\mathrm{CD}^{-}$. According to the criteria proposed by Hans et al (12), DLBCL is categorized into germinal center-type $\mathrm{B}$ cell lymphoma $(\mathrm{GCB})\left(\mathrm{CD} 10^{+}\right.$or CD10 $/ \mathrm{bcl}^{-6}$ (MUM1 $^{-}$) and non-GCB (CD10 $\left./ \mathrm{bcl}^{-6} / \mathrm{MUM1}^{+}\right)$ type. The subtype of the studied DLBCL cases was GCB in 11 cases and non-GCB in 35 cases. Although the number of females and non-GCB cases was higher than that of males and GCB cases, there was no collection bias. Histological specimens were fixed in $10 \%$ formalin and routinely processed for paraffin-embedding. Paraffin-embedded specimens were stored in a dark room at room temperature at the Department of Pathology of Osaka University Hospital, and sectioned (4 $\mu \mathrm{m})$ at the time of staining and stained with H\&E and immunoperoxidase procedure. 
Table I. Characteristics of the 46 DLBCL patients.

No. of patients

\begin{tabular}{lc}
\hline Gender & \\
Male & 19 \\
Female & 27 \\
Location & \\
Nodal & 25 \\
Extranodal & 21 \\
Subtype & \\
GCB & 11 \\
Non-GCB & 35 \\
Stage & \\
I & 18 \\
II & 7 \\
III & 4 \\
IV & 17 \\
Treatment & \\
Chemo-radiotherapy & 10 \\
Chemotherapy alone & 28 \\
Radiotherapy alone & 1 \\
Chemotherapy after surgery & 2 \\
Transplantation & 4 \\
No treatment & 1 \\
Response to therapies & 1 \\
CR & \\
PR/NC & \\
PD & \\
\end{tabular}

CR, complete response; PR, partial response; NC, no change; PD, progressive disease.

Based on physical examination records, surgical notes and pathological assessments of the specimens, the Ann Arbor staging system was applied. Eighteen cases were at stage I, 7 at stage II, 4 at stage III and 17 at stage IV. Ten patients received a combination of radiotherapy and chemotherapy, 28 received chemotherapy only, 2 received chemotherapy after surgery, 4 transplantation (3 auto- and 1 allo-transplantation), 1 received radiation therapy only and 1 received no therapy. The clinical outcome was evaluated according to the Guidelines of the International Workshop to Standardize Response Criteria for non-Hodgkin Lymphoma (13). The characteristics of the patients, such as age, tumor location and stage, in the present study were similar to those in previous reports $(14,15)$. The proportion of male DLBCL patients in general was $\sim 55 \%$, indicating that the proportion of female patients was slightly high in the present study. The study was approved by the Ethics Review Board of the Graduate School of Medicine, Osaka University.

Immunohistochemistry. ALDH1 expression was immunohistochemically examined using the anti-ALDH1 antibody (BD Biosciences, Franklin Lakes, NJ, USA). The sections were incubated with anti-ALDH1 diluted at x100, and subsequently processed using the ChemMate EnVision kit (Dako A/S, Glostrup, Denmark). DAB (Dako) was used as a chromogen.
As the negative control, staining was carried out in the absence of the primary antibody. For immunophenotyping of DLBCL, the following monoclonal antibodies were used: CD20, CD3, Bcl-6, CD10 and MUM1 (Dako; dilution at 1:400, 1:50, 1:50, 1:100 and 1:100, respectively). Stained sections were evaluated independently by two pathologists (S.F. and E.M.).

Double staining of ALDH1 with CD20, fascin and CD68. Double staining of ALDH1 with CD20 (a marker for DLBCL cells), fascin (a marker for DCs; Dako) and CD68 (a marker for macrophages; Dako) was carried out using the EnVision G/2 doublestain system (Dako) according to the manufacturer's protocol. For double staining with CD20, sections were first incubated with the anti-CD20 antibody $(1: 100)$, colored with DAB and, subsequently, ALDH1 expression was detected with Permanent Red (Dako). For double staining with CD68 or fascin, sections were initially incubated with ALDH1, colored with DAB and, subsequently, antigen retrieval was carried out using a Pascal pressurized heating chamber (Dako). After incubation with the anti-CD68 (1:100) or anti-fascin (1:100) antibody, sections were colored with Permanent Red. Since red fluorescence is released from Permanent Red, the red-color signal was detected with a fluorescence microscope (Biozero, Keyence, Osaka, Japan).

Statistical analysis. The Chi-square test was used to analyze the correlation between ALDH1 expression and clinicopathological characteristics of the lymphoma cases. Kaplan-Meier methods were used to calculate the overall survival (OS) rate, and differences in survival curves were evaluated with the log-rank test. p-values of $<0.05$ were considered statistically significant.

\section{Results}

Immunohistochemical findings. The expression of ALDH1 was examined in 46 DLBCL cases. The signals for ALDH1 were detected in two types of cells: cells with ample cytoplasm and fine chromatin and those with reticular structure (Fig. 1A). To confirm the absence of ALDH1 signals in DLBCL cells, double staining of ALDH1 and CD20 was performed; $\mathrm{CD} 20^{+}$large cells (brown-colored cells) did not express ALDH1 (Fig. 1B and C).

Double staining of ALDH1 and CD68. Since ALDH1 ${ }^{+}$cells with ample cytoplasm and fine chromatin morphologically resembled macrophages, the double staining of ALDH1 and CD68, a marker for macrophages, was carried out. ALDH1 ${ }^{+}$ cells with ample cytoplasm and fine chromatin were positive for CD68 (Fig. 2A and B). By contrast, ALDH1 ${ }^{+}$cells with reticular morphology were negative for CD68 (Fig. 2A and B).

Double staining of ALDH1 and fascin. Reticular morphology in $\mathrm{ALDH}^{+}$cells suggested that a portion of the DCs was $\mathrm{ALDH}^{+}$. To confirm this, double staining of ALDH1 and fascin was performed. ALDH1 ${ }^{+}$DC-like cells were positive for fascin (Fig. 2C and D).

Clinical implication of $A L D H 1^{+}$cells in DLBCL. Clinical significance of the presence of $\mathrm{ALDH}^{+}$cells in DLBCL was examined. Seventeen and 7 of 46 cases possessed ALDH1 ${ }^{+}$ 

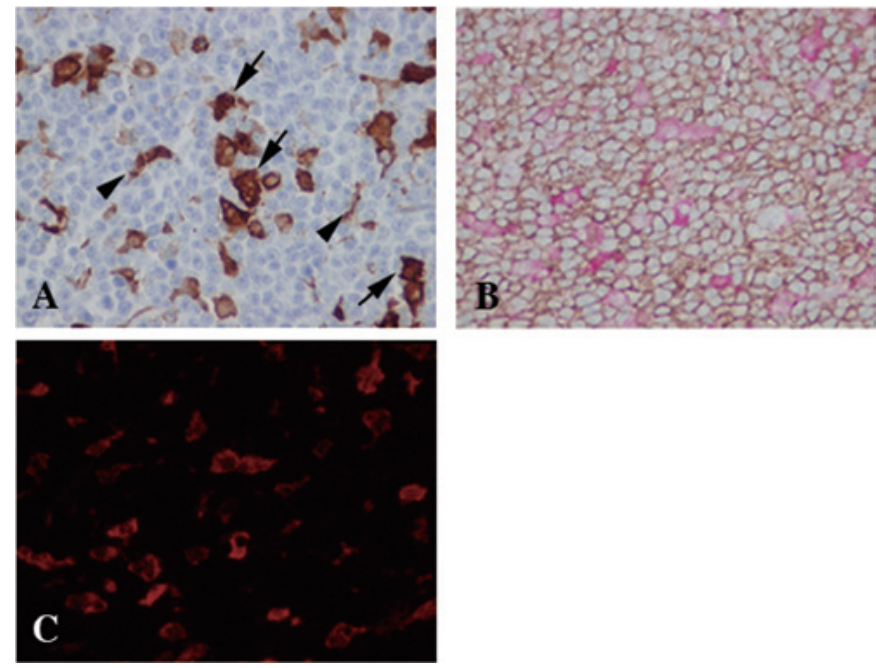

Figure 1. ALDH1 $1^{+}$cells in DLBCL. (A) Two types of cells expressed ALDH1: cells with ample cytoplasm and fine chromatin (arrows) and cells with reticular morphology (arrowheads). (B) Double staining of ALDH1 (red) and CD20 (brown). ALDH1 ${ }^{+}$cells are CD20. (C) Fluorescent image of B. The red-colored $\mathrm{ALDH} 1^{+}$cells are clearly shown.
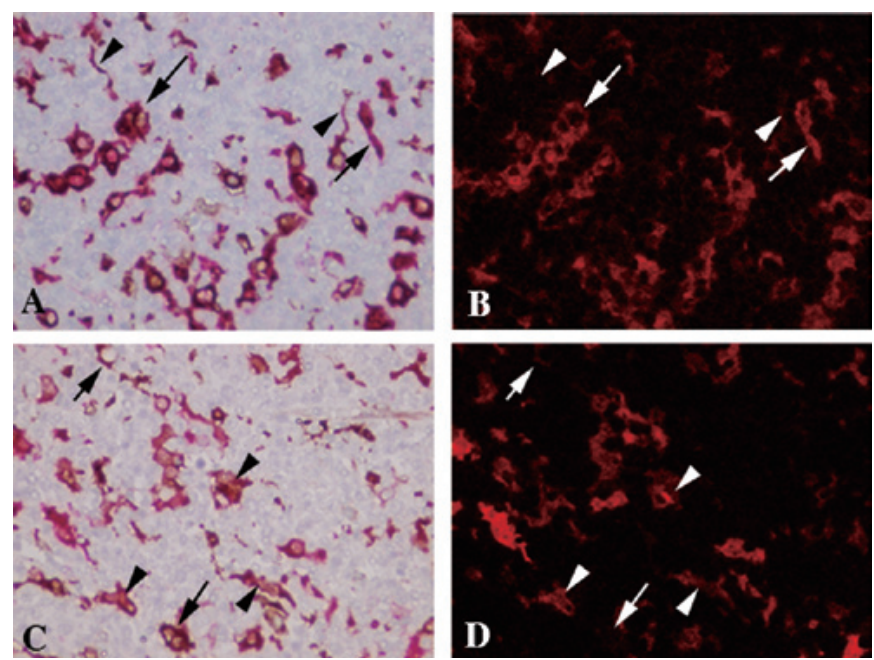

Figure 2. Double staining of ALDH1 and CD68 or fascin. (A) ALDH1 expression is shown in brown and CD68 in red. ALDH1 ${ }^{+}$cells with ample cytoplasm and fine chromatin are positive for CD68 (arrows), whereas $\mathrm{ALDH1}^{+}$cells with reticular morphology are negative for CD68 (arrowheads). (B) Fluorescent image of A. CD68 ${ }^{+}$cells are shown. (C) ALDH1 expression is shown in brown and fascin in red. ALDH1 $1^{+}$cells with reticular morphology are positive for fascin (arrowheads), whereas $\mathrm{ALDH}^{+}$cells with ample cytoplasm and fine chromatin are negative for fascin (arrows). (D) Fluorescent image of $\mathrm{C}$.

macrophages and $\mathrm{ALDH}^{+} \mathrm{DCs}$, respectively. The presence of $\mathrm{ALDH}^{+}$macrophages did not show any correlation with gender, location, subtype, stage of diseases and response to therapies (Table II). By contrast, the presence of $\mathrm{ALDH}^{+}$ DCs was correlated with location $(\mathrm{p}=0.038)$ and response to therapies $(p=0.039)$, although no correlation was detected in gender, subtype and stage of disease (Table III). There was a statistically significant difference in OS between patients with and without $\mathrm{ALDH}^{+} \mathrm{DCs}(\mathrm{p}=0.006)$, although no difference was detected between patients with and without $\mathrm{ALDH} 1^{+}$ macrophages (Fig. 3).
Table II. Correlation between the presence of $\mathrm{ALDH}^{+}$macrophages and clinicopathological parameters.

\begin{tabular}{lccc}
\hline & \multicolumn{2}{c}{ ALDH1 ${ }^{+}$macrophages } & p-value \\
\cline { 2 - 2 } & Absent & Present & \\
\hline Gender & & & 0.544 \\
Male & 11 & 8 & \\
Female & 18 & 9 & \\
Location & & & 0.090 \\
$\quad$ Nodal & 13 & 12 & \\
Extranodal & 16 & 5 & \\
Subtype & & & 0.964 \\
GCB & 7 & 4 & \\
Non-GCB & 22 & 13 & \\
Stage & & & 0.717 \\
I & 10 & 8 & \\
II & 5 & 2 & \\
III & 2 & 2 & \\
IV & 12 & 5 & \\
Response to therapies & & & 0.752 \\
CR & 23 & 13 & \\
PR/NC & 5 & 3 & \\
PD & 1 & 0 & \\
\hline
\end{tabular}

Table III. Correlation between the presence of $\mathrm{ALDH}^{+} \mathrm{DCs}$ and clinicopathological parameters.

\begin{tabular}{lrrr}
\hline & \multicolumn{2}{c}{ ALDH1 ${ }^{+}$DCs } & p-value \\
\cline { 2 - 3 } & Absent & Present & \\
\hline Gender & & & 0.180 \\
Male & 14 & 5 & \\
Female & 24 & 3 & \\
Location & & & 0.038 \\
$\quad$ Nodal & 18 & 7 & \\
Extranodal & 20 & 1 & \\
Subtype & & & 0.938 \\
GCB & 9 & 2 & \\
$\quad$ Non-GCB & 29 & 6 & \\
Stage & & & 0.555 \\
I & 15 & 3 & \\
II & 7 & 0 & \\
III & 3 & 1 & \\
IV & 13 & 4 & \\
Response to therapies & & & 0.039 \\
CR & 32 & 4 & \\
PR/NC & 6 & 2 & \\
PD & 0 & 1 & \\
\hline
\end{tabular}

\section{Discussion}

ALDH1 is reported to be one of the markers for CICs $(1,8,16)$. To search for the presence of CICs in DLBCL, the ALDH1 ${ }^{+}$ 

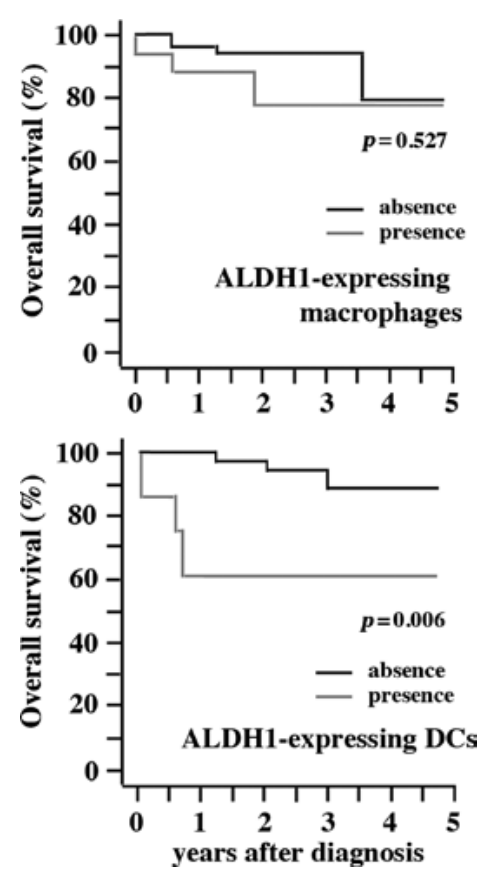

Figure 3. Kaplan-Meier plots. Overall survival (OS) curves of cases with and without $\mathrm{ALDH}^{+}$macrophages or DCs. Cases with $\mathrm{ALDH} 1^{+} \mathrm{DCs}$ showed a less favorable OS.

cells were immunohistochemically evaluated in lymphoid tissues involved by DLCBL. Unexpectedly, there were no $\mathrm{ALDH}^{+}$cells noted among the DLBCL cells. Instead, two types of cells in the stromal tissues expressed ALDH1: one with ample cytoplasm and fine chromatin, and another with reticular morphology. The former was stained with antiCD68, and the latter with anti-fascin, indicating that $\mathrm{ALDH1}^{+}$ cells were macrophages and DCs, respectively.

Recently, ALDH1 expression has been reported in murine DCs derived from the gut (17), lung (5) and dermis (18). In humans, there have been no reports on ALDH1 expression in DCs. To our knowledge, this is the first report on ALDH1 expression in human DCs. Murine gut and dermal ALDH1 ${ }^{+}$ DCs induce regulatory T cells $(18,19)$, and thus may inhibit antitumorigenic immunity. The present study revealed that DLBCL patients with ALDH1 ${ }^{+}$DCs were associated with an unfavorable prognosis compared to those without, suggesting that human $\mathrm{ALDH}^{+} \mathrm{DCs}$ may induce regulatory $\mathrm{T}$ cells and inhibit antitumorigenic immunity. Further studies on the function of human $\mathrm{ALDH}^{+} \mathrm{DCs}$ may clarify their precise role in antitumorigenic immunity.

Recently, Guilliams et al found expression of ALDH1 in macrophages in intestinal lamina propria, but did not report on their function (18). To our knowledge, this is the first report on $\mathrm{ALDH}^{+}$macrophages in humans, but failed to show the clinical implication. The role of ALDH1 in macrophage function may be limited, at least in lymphoid tissues involved by DLBCL.

In conclusion, $\mathrm{ALDH}^{+}$macrophages and $\mathrm{ALDH}^{+} \mathrm{DCs}$ were found in the stromal tissues of DLBCL. Although no clinical implications were found to be associated with the presence of $\mathrm{ALDH}^{+}$macrophages, cases with $\mathrm{ALDH}^{+} \mathrm{DCs}$ demonstrated a less favorable prognosis compared to those without.

\section{Acknowledgements}

The authors thank Ms. Megumi Sugano, Ms. Etsuko Maeno and Ms. Takako Sawamura for the technical assistance. This study was supported by grants from the Ministry of Education, Culture, Sports, Science and Technology, Japan.

\section{References}

1. Ginestier C, Hur MH, Charafe-Jauffret E, et al: ALDH1 is a marker of normal and malignant human mammary stem cells and a predictor of poor clinical outcome. Cell Stem Cell 1: 555-567, 2007.

2. Liu S, Ginestier C, Charafe-Jauffret E, Foco H, Kleer CG, Merajver SD, Dontu G and Wicha MS: BRCA1 regulates human mammary stem/progenitor cell fate. Proc Natl Acad Sci USA 105: 1680-1685, 2008.

3. Ibarra I, Erlich Y, Muthuswamy SK, Sachidanandam R and Hannon GJ: A role for microRNAs in maintenance of mouse mammary epithelial progenitor cells. Genes Dev 21: 3238-3243, 2007.

4. Dave B and Chang J: Treatment resistance in stem cells and breast cancer. J Mammary Gland Biol Neoplasia 14: 79-82, 2009.

5. Jiang F, Qiu Q, Khanna A, Todd NW, Deepak J, Xing L, Wang H, Liu Z, Su Y, Stass SA and Katz RL: Aldehyde dehydrogenase 1 is a tumor stem cell-associated marker in lung cancer. Mol Cancer Res 7: 330-338, 2009.

6. Rasheed ZA, Yang J, Wang Q, et al: Prognostic significance of tumorigenic cells with mesenchymal features in pancreatic adenocarcinoma. J Natl Cancer Inst 102: 340-351, 2010.

7. Su Y, Qiu Q, Zhang X, Jiang Z, Leng Q, Liu Z, Stass SA and Jiang F: Aldehyde dehydrogenase 1 A1-positive cell population is enriched in tumor-initiating cells and associated with progression of bladder cancer. Cancer Epidemiol Biomarkers Prev 19: 327-337, 2010.

8. Li T, Su Y, Mei Y, Leng Q, Leng B, Liu Z, Stass SA and Jiang F: ALDH1A1 is a marker for malignant prostate stem cells and predictor of prostate cancer patients' outcome. Lab Invest 90: 234-244, 2010.

9. Bonnet D and Dick JE: Human acute myeloid leukemia is organized as a hierarchy that originates from a primitive hematopoietic cell. Nat Med 3: 730-737, 1997.

10. Udagawa $\mathrm{T}$ and Wood M: Tumor-stromal cell interactions and opportunities for therapeutic intervention. Curr Opin Pharmacol 10: 369-374, 2010.

11. Ma J, Liu L, Che G, Yu N, Dai F and You Z: The M1 form of tumorassociated macrophages in non-small cell lung cancer is positively associated with survival time. BMC Cancer 10: 112, 2010.

12. Hans CP, Weisenburger DD, Greiner TC, et al: Confirmation of the molecular classification of diffuse large B-cell lymphoma by immunohistochemistry using a tissue microarray. Blood 103: 275-282, 2004.

13. Cheson BD, Horning SJ, Coiffier B, et al: Report of an international workshop to standardize response criteria for nonHodgkin's lymphomas. NCI Sponsored International Working Group. J Clin Oncol 17: 1244-1253, 1999.

14. The Non-Hodgkin's Lymphoma Classification Project: A Clinical Evaluation of the International Lymphoma Study Group Classification of Non-Hodgkin's Lymphoma. Blood 89: 3909-3918, 1997.

15. Armitage JO and Weisenburger DD: New approach to classifying non-Hodgkin's lymphomas: clinical features of the major histologic subtypes. Non-Hodgkin's Lymphoma Classification Project. J Clin Oncol 16: 2780-2795, 1998.

16. Huang EH, Hynes MJ, Zhang T, et al: Aldehyde dehydrogenase 1 is a marker for normal and malignant human colonic stem cells (SC) and tracks SC overpopulation during colon tumorigenesis. Cancer Res 69: 3382-3389, 2009.

17. Saurer L, McCullough $\mathrm{KC}$ and Summerfield A: In vitro induction of mucosa-type dendritic cells by all-trans retinoic acid. J Immunol 179: 3504-3514, 2007.

18. Guilliams M, Crozat K, Henri S, et al: Skin-draining lymph nodes contain dermis-derived CD103(-) dendritic cells that constitutively produce retinoic acid and induce Foxp3(+) regulatory T cells. Blood 115: 1958-1968, 2010.

19. Coombes JL and Powrie F: Dendritic cells in intestinal immune regulation. Nat Rev Immunol 8: 435-446, 2008. 